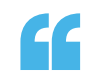

\section{For some time we have been thinking about the connection between topological bulk and surface states and catalysis}

\title{
Topology does the water splits
}

Topological Weyl semimetals show promise as a new class of efficient catalysts for the hydrogen evolution reaction, as Claudia Felser, C. N. R. Rao and co-workers report in Advanced Materials.

The development of more efficient catalysts is not only important for various industrial processes, but also essential for the viability of clean energy technologies. For example, water splitting, in which water molecules are photo- and/or electrochemically split into molecular oxygen and hydrogen, could underpin a clean hydrogen economy. A range of materials has been used for the catalysis of water splitting, but activities are still much lower than required. It is evident that designing improved catalytic materials for future technology requires a better understanding of this process at a fundamental level, and possibly a fresh angle on the problem. "In our new approach we look at parts of the reaction path through the lens of topology," says Felser.

The researchers hypothesized that topological materials (that is,

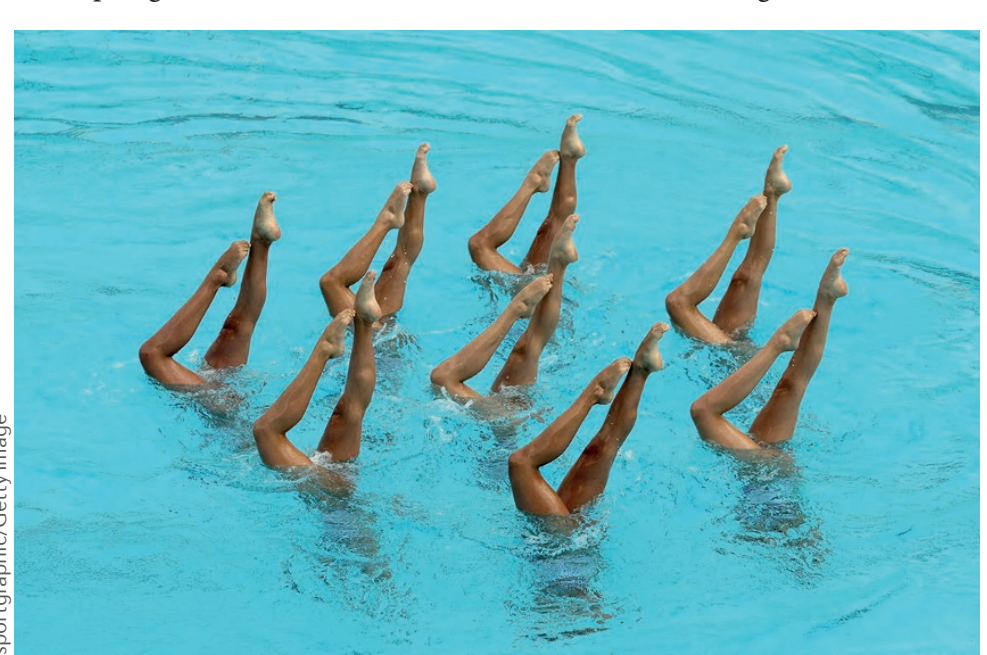

materials with different bulk and surface properties) could produce superior catalysts because they have protected surface states that are robust against scattering and defects, which could be helpful for reactions taking place at their surface. "For some time we have been thinking about the connection between topological bulk and surface states and catalysis," remarks Felser, whose team has previously demonstrated that gold and platinum, both recognized as good catalysts, have such protected topological surface states. They proposed that the occurrence of these states is related to a geometrical property of the band structure of the metals.

In their new experiments, the team concentrated on Weyl semimetals, which have the type of band structure that leads to protected surface states. Additionally, the desirable high charge-carrier mobility is guaranteed by the linear dispersion of the band structure around the points where the valence and conduction bands touch. These are the Weyl cones, the 3D analogues of the Dirac cones in graphene, which give this class of materials its name. Using the hydrogen evolution reaction as a model reaction, the researchers compared the catalytic performance of a conventional metallic transition metal dichalcogenide with that of a topological one. Although both materials are expected to be good catalysts from purely thermodynamic considerations, the conventional dichalcogenide was completely inactive, whereas the topological material proved to be an excellent catalyst.

These results encouraged them to cast a wider net and investigate the catalytic performance of several transition metal monopnictides, in which topological surface states have been directly observed. All generally showed high catalytic activity, and the researchers were able to correlate the relative performance with the free energy of hydrogen absorption on the surface of the materials. Following on from these findings for one family of topological semimetals, the team plans to generalize the concept to help identify the best catalysts for specific reactions. "Topology is a new and rapidly expanding field in condensed matter physics and materials science that could also have an important impact in chemistry," concludes Felser; however, further research into surface states is needed to clarify the role of topology in catalysis.

Nina Meinzer, Associate Editor, Nature Communications

ORIGINAL ARTICLE Rajamathi, C. R. et al. Weyl semimetals as hydrogen evolution catalysts. Adv. Mater. http://dx.doi.org/10.1002/adma.201606202 (2017)

FURTHER READING Yan, B. et al. Topological states on the gold surface. Nat. Commun. 6, 10167 (2015) 